Manfred G. Schmidt

\title{
Sozialpolitik als Stabilisierungsfaktor der bundesrepublikanischen Entwicklung?"
}

Wirkt die Sozialpolitik als Stabilisierungsfaktor der Politik, der Gesellschaft und der Wirtschaft in Deutschland? Diese Frage wird in der politischen und der fachwissenschaftlichen Diskussion höchst unterschiedlich beantwortet. Die Spannweite zwischen den Auffassungen ist groß. Sie reicht von der These der generellen Stabilisierung durch Sozialpolitik und der Meinung, die Sozialpolitik sei eine Funktionsvoraussetzung stabiler demokratischer Entwicklung und leistungsfähiger Wirtschaft und Gesellschaft, bis zur These der Unterminierung von Wirtschaft und Gesellschaft durch ein engmaschiges Netz der sozialen Sicherung.

Dass die Antworten so unterschiedlich ausfallen, hängt teilweise mit den politischweltanschaulichen Positionen der Beobachter zusammen. Ein radikaler Wirtschaftsliberaler beispielsweise wird eine weit ausgebaute Sozialpolitik, wie in Deutschland, eher skeptisch beurteilen und eher auf Instabilität tippen. Ein Fürsprecher der Linken hingegen wird das Problem nicht in zu viel Sozialpolitik sehen, sondern in zu wenig sozialer Sicherung, und er wird von mehr Sozialpolitik mehr Stabilität erwarten.

Die unterschiedlichen Antworten auf die Frage nach den Stabilisierungswirkungen der Sozialpolitik wurzeln aber auch in unterschiedlichen wissenschaftlichen Ansätzen, in der Auswahl der untersuchten Fälle und in der Fachdisziplin der Wissenschaftler. Die meisten Wirtschaftswissenschaftler neigen zu einer skeptischen Sicht auf die Stabilisierungsthese, während ein beträchtlicher Teil der Sozialpolitikfachleute unter den Soziologen, Politikwissenschaftlern und Historikern eher signifikante Stabilisierungsleistungen zumindest in politischer und meistens auch in gesellschaftlicher Hinsicht verortet. ${ }^{1}$

Besonders belastbare Befunde zu Stabilisierungs- oder Destabilisierungseffekten der Sozialpolitik sind, so lehrt der bisherige Forschungsstand, am ehesten von Beobachtungsperspektiven zu erwarten, die - erstens - den internationalen und historischen Vergleich zur Abschätzung der Wirkung der Sozialpolitik heranziehen, zweitens die Ergebnisse von historiographischen Wirkungsanalysen berücksichtigen, ${ }^{2}$ drittens transdisziplinär angelegt sind und - viertens - bei der Abwägung von Für und Wider sowohl die

* Überarbeitete Fassung des Vortrages vor der Generalversammlung der Görres-Gesellschaft in Salzburg am 28.9.2009.

1 Vgl. als Überblick zur Diskussion Manfred G. Schmidt / Tobias Ostheim / Nico A. Siegel / Reimut Zohlnhöfer (Hg.), Der Woblfahrtsstaat, Wiesbaden, S. 410-422.

2 Ein mustergültiges Beispiel ist Hans-Günter Hockerts, Sozialpolitische Entscheidungen im Nachkriegsdeutschland. Alliierte und deutsche Sozialversicherungspolitik 1945 bis 1957, Stuttgart 1980.

ZfP 56. Jg. 4/2009 
Vorzüge und Nachteile der Sozialpolitik wie auch ihren Nutzen und ihre Kosten erörtern, und zwar in politischer, gesellschaftlicher und wirtschaftlicher Hinsicht. ${ }^{3}$

Just auf dieser Perspektivenbasis und der Grundlage des andernorts ausführlicher dokumentierten Forschungsstandes ${ }^{4}$ gründet der vorliegende Beitrag. Er gliedert sich in vier Teile. Zunächst wird die für die Frage zentrale unabhängige Variable - die Sozialpolitik in der Bundesrepublik Deutschland - in ihren wichtigsten Strukturen und aus dem Blickwinkel der Investitionen in den Sozialstaat porträtiert. Anschließend werden die Stärken der Sozialpolitik sowie ihre Nebenwirkungen und Folgeprobleme bilanziert, und zwar jeweils in politischer, gesellschaftlicher und wirtschaftlicher Hinsicht und unter Berücksichtigung des Leistungsprofils und der Akzeptanz der Sozialpolitik in der Bevölkerung. Der letzte Abschnitt fasst die Ergebnisse zusammen. Dort wird dargelegt, dass die Sozialpolitik zweiwertig wirkt: sie ist zugleich Problemlöser und Problemerzeuger. Zudem wächst die Lücke zwischen den umfänglichen Investitionen in die Sozialpolitik sowie ihren Leistungen einerseits und ihrer tendenziell abnehmenden Akzeptanz andererseits. Das deutet auf einen abnehmenden politischen Grenznutzen der Sozialpolitik hin.

3 Vgl. insbesondere neben Jens Alber, »Hat sich der Wohlfahrtsstaat als soziale Ordnung bewährt? « in: Karl-Ulrich Mayer (Hg.), Die beste aller Welten? Marktliberalismus versus Woblfabrtsstaat, Frankfurt a.M. 2001, S. 59-112; Stephan Leibfried / Rainer Müller / Winfried Schmähl / Manfred G. Schmidt, »Thesen zur Sozialpolitik in Deutschland « in: Zeitschrift für Sozialreform 44, Nr. 8 (1998), S. 525-569.

4 Die derzeit ausschlussreichsten Quellen zu dieser Frage finden sich für den Zeitraum von 1945 bis Mitte der 1990er Jahre in: Bundesministerium für Arbeit und Soziales / Bundesarchiv (Hg.), Geschichte der Sozialpolitik in Deutschland seit 1945, 11 Bände, Baden-Baden, 2001-2008, insbesondere in den Bänden 1-7 und 11. Es sind dies im Einzelnen: Bundesministerium für Arbeit und Sozialordnung / Bundesarchiv (Hg.), Grundlagen der Sozialpolitik. Band 1, Baden-Baden 2001; Udo Wengst (Bandherausgeber), 1945-1949. Die Zeit der Besatzungszonen Sozialpolitik zwischen Kriegsende und der Gründung zweier deutscher Staaten. Band 2, Baden-Baden 2001; Günther Schulz (Bandherausgeber), Bundesrepublik Deutschland 1949-1957. Bewältigung der Kriegsfolgen, Rückkehr zur sozialpolitischen Normalität, Band 3, Baden-Baden 2006; Michael Ruck / Marcel Boldorf (Bandherausgeber), Bundesrepublik Deutschland 1957-1966. Sozialpolitik im Zeichen des erreichten Woblstands, Band 4, Baden-Baden 2008; Hans Günter Hockerts (Bandherausgeber), Bundesrepublik Deutschland 1966-1974. Eine Zeit vielfältigen Aufbruchs, Band 5, Baden-Baden 2007; Martin H. Geyer (Bandherausgeber), Bundesrepublik Deutschland 1974-1982. Nene Herausforderungen, wachsende Unsicherbeit, Band 6, Baden-Baden 2008; Manfred G. Schmidt (Bandherausgeber), Bundesrepublik Deutschland 1982-1989. Finanzielle Konsolidierung und institutionelle Reform, Band 7, Baden-Baden 2005; Gerhard A. Ritter (Bandherausgeber), Bundesrepublik Deutschland 1989-1994. Sozialpolitik im Zeichen der Vereinigung, Band 11, Baden-Baden 2007. Zur Einführung und als Überblick über dieses Großunternehmen vgl. Manfred G. Schmidt, Einleitung - zugleich eine Einführung zur »Geschichte der Sozialpolitik in Deutschland seit 1945 in: Zeitschrift für Sozialreform 55, Nr.1 (2009), S. 3-9. Bei der Schätzung der Stabilisierungseffekte der Sozialpolitik stützt sich der Verfasser des vorliegenden Essays insbesondere auch auf die Befunde in Gerhard A. Ritter, Der Preis der deutschen Einheit: Die Wiedervereinigung und die Krise des Sozialstaates, München 2006; Manfred G. Schmidt, Sozialpolitik in Deutschland. Historische Entwicklung und internationaler Vergleich, Wiesbaden 2005, 3. Auflage, Teil 3 sowie Schmidt u.a., Wohlfahrtsstaat (Anm. 1). 


\section{Strukturen der Sozialpolitik in der Bundesrepublik Deutschland}

Deutschlands Sozialpolitik verkörpert den „Sozialversicherungsstaat “ ${ }^{5}$ so heißt es. Das ist nicht falsch, aber unvollständig. Sicherlich finanzieren die Sozialbeiträge der versicherten Arbeitnehmer und ihrer Arbeitgeber fast 60 Prozent des gesamten Sozialbudgets. Doch rund 40 Prozent des Sozialbudgets stammen aus überwiegend steuerfinanzierten Quellen und werden nicht nach dem Prinzip der Sozialversicherung verausgabt, sondern folgen anderen Grundsätzen wie Alimentation, Entschädigung, Fürsorge und soziale Hilfe. ${ }^{6} \mathrm{Zu}$ diesen Systemen der sozialen Sicherung zählen beispielsweise die beamtenrechtliche Fürsorge, die Kriegsopferversorgung, der Lastenausgleich und die diversen Mindestsicherungssysteme, die derzeit für rund 10 Prozent der Wohnbevölkerung in Deutschland die wichtigste Finanzierungsquelle des Lebensunterhaltes sind. ${ }^{7}$ Eine Vielzahl von Risiken deckt die Sozialpolitik ab und erfasst dabei mittlerweile fast die gesamte Wohnbevölkerung. Ergänzend kommt ein weites Feld der Arbeitspolitik hinzu: Die Sozialpolitik greift tief in die Arbeitswelt ein, insbesondere beim Arbeitsschutz und bei der - auch im internationalen Vergleich - weit ausgebauten Arbeitnehmermitbestimmung. Zur deutschen Sozialpolitik gehört ferner die Delegation wichtiger Aufgaben an die Tarifparteien, so in der Lohnpolitik, und an die Wohlfahrtsverbände.

Der Wiederaufbau der Sozialpolitik nach dem Krieg und ihr Ausbau haben in der Bundesrepublik Deutschland einen der weltweit aufwendigsten Wohlfahrtstaaten geschaffen, ${ }^{8}$ der nahezu allen Staatsbürgern im Schadens- oder Notfall Sozialleistungen zuteilt, und zwar als Staatsbürgerrecht, nicht als Almosen. Finanziert werden die Systeme der sozialen Sicherung aus einem umfänglichen Sozialbudget. Es umfasst laut neuester Schätzung des Bundesarbeitsministeriums im Jahre 2009754 Milliarden Euro oder 31,9 Prozent des Bruttoinlandsproduktes ${ }^{9}$ - ein Vielfaches der Finanzausstattung jedes anderen Politikfeldes im Lande.

\section{Wirkungen: Politische, gesellschaftliche und wirtschaftliche Stabilisierungsfunktionen der Sozialpolitik}

Ein Politikfeld wie die Sozialpolitik, in das mittlerweile mehr als 750 Milliarden Euro jährlich investiert werden, hat naturgemäß gewaltige Wirkungen. Zu den besonders berichtenswerten Wirkungen gehört diese: die Sozialpolitik bewältigt ihre ureigenen Aufgaben insgesamt gut. Sie verhindert den Absturz in materielle Verelendung. Anders als

5 Sven Jochem, »Reformpolitik im deutschen Sozialversicherungsstaat « in: Manfred G. Schmidt (Hg.), Woblfahrtsstaatliche Politik. Institutionen, politischer Prozess und Leistungsprofil, Opladen 2001, S. 193-261.

6 So der Stand 2009 (Quelle: Bundesministerium für Arbeit und Soziales (BMAS), Sozialbericht 2009, Bonn 2009, Tab. I-1, S. T1).

7 Statistisches Bundesamt u.a. (Hg.), Datenreport 2008. Ein Sozialbericht für die Bundesrepublik Deutschland, Wiesbaden 2008.

8 Schubert, Klaus / Hegelich, Simon/Bazant, Ursula (Hg.), Europäische Woblfahrtssysteme, Wiesbaden 2009.

9 BMAS, Sozialbudget 2009, Tab. I-1.

ZfP 56. Jg. 4/2009 
in den USA kann man hierzulande nicht gleichsam von der Ersten Welt in die Dritte Welt fallen. ${ }^{10}$ Die Sozialpolitik in Deutschland schützt zudem relativ zuverlässig gegen Not infolge von Einkommensausfall durch Alter, Arbeitslosigkeit, Invalidität, Krankheit, Pflegefallabhängigkeit und Tod des Ernährers, um nur die aufwendigsten Risikofelder zu benennen. Dass krasse soziale Ungleichheit in Deutschland beträchtlich vermindert wird, hängt ebenfalls mit der Sozialpolitik zusammen. Davon zeugt etwa die Verminderung der potenziellen Armutsquote allein durch Sozialtransfers: Diese reduzieren den Anteil der in relativer Einkommensarmut lebenden Bevölkerung in Deutschland von 26 auf 13 Prozent - auch im internationalen Vergleich ein herausragendes Ergebnis der Armutsbekämpfung. ${ }^{11}$

$\mathrm{Zu}$ den großen Stabilisierungserfolgen der Sozialpolitik wird man außerdem zählen können, dass sie die Politik gegen Erschütterungen infolge schwerer Wirtschaftskrisen abschirmt. Mehr noch: Die Sozialpolitik wirkt in solchen Fällen buchstäblich wie ein „Problemzerstäuber“: ${ }^{12}$ Sie zerlegt massenhaft auftretende Probleme in kleinste, sich nicht zu einer kompakten Problemmasse formierende Teile: den einen behandelt sie als Fall der Frühverrentung, den anderen als Fall der Erwerbsminderung, den dritten als Kurzarbeiter, den vierten als Arbeitslosengeldbezieher. Andere wiederum kommen als Arbeitslosengeld II-Empfänger in den Hoheitsbereich des Sozialstaates oder als Adressaten von Sozialhilfe, Sozialgeld oder Wohngeld, um nur einige Beispiele zu erwähnen. Überdies zählt zu den politisch stabilisierenden Wirkungen der Sozialpolitik dies: Sie ist eine potenziell zentrale Quelle der Legitimation im demokratischen Staat. Sie wirkt durch sogenannte „Output-Legitimität“, also durch Anerkennungswürdigkeit von Leistung, in diesem Fall von sozialpolitischer Leistung. Dieser Mechanismus spielte in der Politik zur deutschen Einheit und bei der Integration der Bevölkerung der neuen Bundesländer seit der Einheit eine zentrale Rolle. ${ }^{13}$ Gutzuschreiben ist der Sozialpolitik ferner, dass sie zur Linderung großer gesellschaftlicher Spannungen beiträgt: Sie entschärft Konflikte etwa zwischen wirtschaftlich wohlhabenden Regionen und wirtschafts- und finanzschwachen Gebieten: Letztere profitieren von Sozialleistungen in überdurchschnittlichem Maße. Zudem dämmt die Sozialpolitik den Konflikt zwischen Arbeit und Kapital ein. Ihn entschärft die Sozialpolitik unter anderem dadurch, dass die sozialen Transferzahlungen der markterzeugten sozialen Ungleichheit den Stachel nehmen, sowie

10 Der entscheidende Schutzwall gegen diesen Absturz sind in Deutschland die differenzierten, insgesamt leistungsfähigen Mindestsicherungssysteme wie etwa Arbeitslosengeld II, Sozialgeld, Sozialhilfe, Wohngeld und Kriegsopferentschädigung, um nur einige Wirkmechanismen zu erwähnen. In den USA fehlt ein solcher Grundsicherungsschutz.

11 Lebenslagen in Deutschland. Der 3. Armuts- und Reichtumsbericht der Bundesregierung, Berlin 2005, S. IX-XII. Gemessen wird die Armutsrisikoschwelle in diesem Falle durch 60 Prozent des mittleren Äquivalenzeinkommens.

12 Roland Czada, »Der Kampf um die Finanzierung der deutschen Einheit« in: Gerhard Lehmbruch (Hg.), Einigung und Zerfall. Deutschland und Europa nach dem Ende des Ost-WestKonflikts. 19. Wissenschaftlicher Kongreß der Deutschen Vereinigung für Politische Wissenschaft, Opladen 1995, S. 73-102.

13 Vgl. Ritter, Der Preis der Einheit (Anm. 4). Allerdings war dafür, so Ritter weiter, ein hoher Preis zu entrichten: die Verschärfung der Krise des Sozialstaats. 
dadurch, dass die Sozialpolitik die Sozialstruktur durch Kombinationen von Markt- und Transfereinkommen differenziert und der Herausbildung klassenhomogener Interessenlagen entgegenwirkt. Die Sozialpolitik vermindert ökonomische Spannungslinien zudem dadurch, dass die Entscheidungen über die meisten Sozialeinkommen im Parlament getroffen werden, nicht in den Betrieben. Das entlastet die Unternehmen von heftigem Streit über einen beträchtlichen Teil der Arbeitskosten. Potenziell brisante Verteilungsfragen werden auf diese Weise nicht zu Verfassungsfragen. Sie werden vielmehr auf verschiedene Arenen aufgeteilt, fragmentiert und kleingearbeitet.

Dies und etliche andere Wirkungen, wie die Stabilisierung der Lebenslage von Personen im Rentenalter, summieren sich zu einer beachtlichen Leistung der Sozialpolitik in Deutschland. Das honoriert ein Großteil der Bevölkerung. Nach wie vor genießt die Sozialpolitik „eine hohe Akzeptanz" 14 bei der großen Mehrheit der Bevölkerung und eine insgesamt relativ hohe Zufriedenheit. Mehr noch: Die meisten Befragten befürworten sogar höhere Sozialausgaben für fast alle größeren Sozialpolitikfelder mit Ausnahme der Arbeitslosigkeit. ${ }^{15}$

Beachtlich sind auch die ökonomischen Stabilisierungsfunktionen der Sozialpolitik. Das überrascht angesichts der heftigen Kritik an der Sozialpolitik aus dem Munde vieler Wirtschaftswissenschaftler. Doch vielen Ökonomen entgeht der beträchtliche „wirtschaftliche Wert"16 der Sozialpolitik. Sie entlastet - wie schon erwähnt - Betriebe von gefährlichen Konflikten über das Sozialeinkommen. Auch protegiert sie den Faktor Arbeitskraft. Das ist im Falle hochqualifizierter Arbeitskräfte eine besonders wichtige Funktion und ist angesichts der Bedeutung des dort angehäuften „Humankapitals“ auch für das gesamtwirtschaftliche Wachstum von großer Bedeutung. ${ }^{17}$

Aufgrund ihrer hohen Kosten für die Wirtschaft wirkt die Sozialpolitik zudem als Anreiz für produktivitätssteigernde, arbeitssparende Investitionen. Sie ist insofern in ökonomischer Hinsicht, man verzeihe das harte Wort, eine Modernisierungspeitsche, die die Unternehmen zum arbeitssparenden technischen Fortschritt antreibt. Dass die Sozialpolitik zudem die gesamtwirtschaftliche Nachfrage stabilisieren kann, erweist sich namentlich in wirtschaftlichen Rezessionen als Vorteil, so auch in der Finanzmarkt- und Realwirtschaftskrise von 2008 und 2009. Ferner muss eine weit ausgebaute Sozialpolitik nicht mit dem Ziel einer dynamischen wirtschaftlichen Entwicklung kollidieren. Vielmehr kommt es insbesondere darauf an, ob die Sozialpolitik und obendrein die gesamte

14 Statistisches Bundesamt u.a. (Hg.), Datenreport 2008 (Anm. 7), S. 294.

15 Statistisches Bundesamt u.a. (Hg.), Datenreport 2008 (Anm. 7), S. 292.

16 Götz Briefs, »Der wirtschaftliche Wert der Sozialpolitik « in: Gesellschaft für Sozialreform (Hg.), Die Reform des Schlichtungswesens. Der wirtschaftliche Wert der Sozialpolitik. Bericht über die Verhandlungen der XI. Generalversammlung der Gesellschaft für Soziale Reform in Mannheim am 24./25. Oktober 1929, Jena, 1930, S. 140-170; Georg Vobruba (Hg.) 1989: Der wirtschaftliche Wert der Sozialpolitik, Berlin - New York.

17 Peter H. Lindert, Growing Public. Social Spending and Economic Growth since the Eighteenth Century, 2 Bde., Cambridge 2004; Torben Iversen, Capitalism, Democracy and Welfare, Cambridge 2005 . 
Steuerpolitik beschäftigungs- und wachstumsfreundlich angelegt ist. So der Befund eines gründlichen internationalen und wirtschaftshistorischen Vergleichs. ${ }^{18}$

\section{Politische, soziale und ökonomische Nebenwirkungen und Folgeprobleme einer weit ausgebauten Sozialpolitik}

Allerdings hat die Sozialpolitik nicht nur Probleme gelöst, sondern auch Probleme geschaffen. Sie ist demnach doppelwertig: sie ist zugleich Problemlöser und Problemerzeuger. $\mathrm{Zu}$ den von ihr hervorgebrachten Problemen gehören etwa sozialstaatsinduzierte Arbeitsmarktverwerfungen, ${ }^{19}$ beispielsweise die Verfestigung des Anteils der Langzeitarbeitslosen an allen Arbeitslosen. Zu den politischen Kosten der Sozialpolitik zählt ferner der Zentralisierungs- und Bürokratisierungsschub, den sie mit sich bringt. Und insbesondere in Staaten mit mittlerer gesamtwirtschaftlicher Abgabenquote - wie in der Bundesrepublik Deutschland - wirkt ein aufwändiges Sozialbudget als Finanzierungsbremse für andere ausgabenintensive Aufgabenfelder, ja als Mechanismus der Verdrängung oder Blockierung von finanziell aufwändiger Daseinsvorsorge jenseits der Sozialpolitik. ${ }^{20}$ Die Unterfinanzierung des deutschen Bildungswesens beispielsweise ist ohne den Verdrängungseffekt des umfänglichen Sozialbudgets (und die politisch leichtere Durchsetzbarkeit der Sozialstaatsfinanzierung) nicht zu verstehen. ${ }^{21}$

Einiges spricht für die These, dass diese Verdrängungseffekte nicht kleiner, sondern eher größer werden. Das stärkste Argument hierfür ist die Beobachtung, dass die Wohlfahrtsstaatsklientel (im Sinne aller Personen, die ihren Lebensunterhalt überwiegend aus Sozialeinkommen finanzieren) heute schon rund 40 Prozent der Wahlberechtigten ausmacht und allein aufgrund der Alterung der Gesellschaft noch weiter zunehmen wird. ${ }^{22}$

Auch die gesellschaftlichen Nebenwirkungen und Folgeprobleme der Sozialpolitik in Deutschland sind beachtlich. Zu ihnen gehört der „Wohlfahrtskorporatismus“, 23 in den sich die Sozialpartner im Schatten einer weit ausgebauten Sozialpolitik verstricken. Wohlfahrtskorporatismus meint eine Staat-Verbände-Beziehung, die sich von der angebots- und nachfrageseitigen Variante des Korporatismus der 1970er Jahre durch die enge Verknüpfung von Sozialpolitik und Arbeitsbeziehungen unterscheidet und dadurch,

18 Lindert, Growing Public (Anm. 16).

19 Wolfgang Franz, Arbeitsmarktökonomik, Berlin u. Heidelberg 2006 (6. Aufl.).

20 Francis G. Castles (Hg.), The Disappearing State. Retrenchment Realities in an Age of Globalisation, Cheltenham - Northampton 2007.

21 Manfred G. Schmidt, »Die öffentlichen und privaten Bildungsausgaben in Deutschland im internationalen Vergleich « in: Zeitschrift für Europa- und Staatswissenschaften 2, Nr. 1 (2004), S. 7-31; Frieder Wolf, Bildungsfinanzierung in Deutschland, Wiesbaden 2008.

22 Zur Berechnung Manfred G. Schmidt 2008, »Die Sozialpolitik der zweiten Großen Koalition (2005 bis 2009) « in: Christoph Egle / Reimut Zohlnhöfer (Hrsg.), Die zweite Große Koalition, Wiesbaden 2010 (i.E.). Die Prozentangabe bezieht sich auf die wahlberechtigten Mitglieder der Wohlfahrtsstaatsklientel.

23 Wolfgang Streeck, »Industrial Relations: From State Weakness as Strength to State Weakness as Weakness. Welfare Corporatism and the Private Use of the Public Interest « in: Simon Green / William E. Paterson (Hg.), Governance in Contemporary Germany, Cambridge 2005. 
dass die Kosten der korporatistischen Arbeitsbeziehungen (einschließlich der dort gepflegten Hochlohnpolitik) auf die Sozialpolitik abgewälzt werden, beispielsweise auf die Arbeitslosenversicherung oder über Frühverrentungsarrangements auf die Rentenversicherung. Der Lehre vom Wohlfahrtskorporatismus zufolge haben die Sozialpartner den Sozialstaat als funktionales Äquivalent zum Keynesianismus zu nutzen verstanden. Somit können sie beschäftigungsschädliche Wirkungen ihrer Tarifpolitik kompensieren, und zwar nicht durch Steigerung der gesamtwirtschaftlichen Nachfrage, wie im klassischen Keynesianismus, sondern durch Frühverrentung und andere arbeitsmarktpolitische Maßnahmen.

Zugrunde liegt eine Wirkungskette, die veranschaulicht, wie die Sozialpolitik mittlerweile nicht nur die Schwachen der Gesellschaft protegiert, sondern in besonderem Maße auch den „Insidern“ des Arbeitsmarktes zugute kommt: den „Arbeitsplatzbesitzern“. Die Sozialpolitik stärkt den arbeits- und sozialrechtlichen Schutzwall um die Arbeitsplatzbesitzer und die Gewerkschaften und befestigt deren Position im Streit um Lohnund Arbeitsbedingungen. Das spiegeln auch die Daten zum arbeitsrechtlichen Schutz der Beschäftigung wider. Dieser ist im Primärarbeitsmarkt, also bei arbeits- und sozialrechtlich gut abgesicherten Beschäftigungsverhältnissen, umso höher, je weiter ausgebaut die Sozialpolitik ist. ${ }^{24}$ Auf diese Weise werden die Beschäftigten im Primärarbeitsmarkt gleich zweifach Nutznießer des Sozialstaates: als ein Hauptadressat der sozialen Sicherung und als Hauptgewinner der durch Sozialpolitik und Arbeitsrecht befestigten Position der Tarifpartner. Der doppelte Schutzwall aber verleitet die Arbeitsplatzbesitzer und ihre Interessenverbände sowie kooperationsbereite Arbeitgeber häufig dazu, Tarifverhandlungen zu Lasten Dritter abzuschließen, beispielsweise zu Lasten von Arbeitslosen oder auf Kosten anderer Abgabenzahler, wie etwa im Falle der Frühverrentung durch Abwälzung der Kosten auf die Kassen der Rentenversicherung. ${ }^{25}$

Auch die wirtschaftlichen Folgeprobleme einer weit ausgebauten Sozialpolitik sind gewichtig. Je ehrgeiziger eine Sozialpolitik angelegt ist, desto wahrscheinlicher verstrickt sie sich in den Zielkonflikt zwischen Sozialschutz einerseits und ökonomischer Effizi$e^{e n z^{26}}$ sowie dynamischer Beschäftigung andererseits. Dass Sozialversicherungssysteme wie alle Versicherungen auch eine offene Flanke zum Missbrauch haben, gehört ebenfalls zu den Nebenwirkungen. $\mathrm{Zu}$ den Nebenwirkungen einer weit ausgebauten Sozialpolitik zählt überdies der Anreiz zur Abwanderung in die Schattenwirtschaft. ${ }^{27}$ Zudem wirkt die Finanzierung eines größeren Teils der deutschen Sozialpolitik aus Sozialbeiträgen wie eine Sondersteuer auf den Faktor Arbeit - ein Problem, das durch die Finanzierungsweise der deutschen Einheit, die zu einem Teil über die Erhöhung der Sozialbeitragssätze erfolgte, vergrößert wurde. Die Sondersteuer hat zwar den Vorteil des Anreizes zum arbeitssparenden technischen Fortschritt, sie dämpft aber die Beschäftigung

24 OECD (Organisation for Economic Cooperation and Development), Employment Outlook 2007, Paris 2007.

25 David Rueda, Social Democracy inside out. Partisanship E Labor Market Policy in Industrialized Democracies, Oxford 2007.

26 Arthur Okun, Equality and Efficiency. The Big Tradeoff, Washington D.C. 1975.

27 Friedrich Schneider / Dominik H. Enste, The Shadow Economy, Cambridge 2007. 
mitunter so sehr, dass ein wesentlicher Teil der Einnahmenbasis der Sozialpolitik chronisch defizitär wird.

$\mathrm{Zu}$ den Nebenwirkungen und Folgeproblemen einer weit ausgebauten Sozialpolitik gehört außerdem ihr abnehmender Nutzen für die Sozialpolitikarchitekten. Deutschlands Sozialpolitik laboriert mittlerweile, so zeigen verschiedene neuere Umfragen, an rückläufiger Akzeptanz und abnehmender Zufriedenheit. Gewiss gibt die Bevölkerung insgesamt der Sozialpolitik nach wie vor viel Rückhalt. Allerdings sind Zufriedenheit bzw. Unzufriedenheit je nach Gruppe und je nach Periode verschieden. Erheblich mehr Unzufriedenheit mit der Sozialpolitik besteht generell bei einkommensschwächeren Bevölkerungsgruppen, etwa beim untersten Einkommensquintil, ferner bei Arbeitslosen, bei Geringqualifizierten, bei den Bürgern der neuen Bundesländer insgesamt und bei den 35-Jährigen bis 59-Jährigen, also bei den Hauptzahlern und Leistungsträgern der Sozialpolitik. ${ }^{28}$ Hinzu kommt eine seit der Wiedervereinigung deutlich abnehmende Zufriedenheit mit der Sozialpolitik, zunächst in Westdeutschland und seit Ende der 1990er Jahre auch in Ostdeutschland. So sinkt etwa die anhand einer Zehnerskala gemessene Zufriedenheit mit der Sozialpolitik in Westdeutschland von einem Mittelwert von 7,0 auf 5,5 im Jahre 2006. ${ }^{29}$

Die tendenziell rückläufige Akzeptanz und die tendenziell abnehmende Zufriedenheit haben viele Ursachen. Zu den Hauptbeweggründen wird man vor allem Fünferlei zählen können. Zugrunde liegt ein hohes Tempo des wirtschaftlichen, des technologischen und des sozialen Wandels, das ein höheres Maß an Unkalkulierbarkeit mit sich bringt und insbesondere schwächere Gruppen in der Bevölkerung verunsichert, ja: verängstigt. Hinzu kommt der Sanierungsbedarf der Sozialpolitik, der Umbau- und Rückbaumaßnahmen erforderlich macht, die für die Betroffenen in der Regel spürbar sind und zugleich in der Öffentlichkeit so dramatisiert und in der Regel skandalisiert werden, dass Betroffene und viele Nichtbetroffene die Sanierungsmaßnahmen als eine „Politik der Zumutungen“ wahrnimmt, die mit der liebgewonnenen „Politik des Verteilens“ verglichen und für nicht akzeptabel befunden wird. ${ }^{30}$

\section{Schlussfolgerung}

Die vorliegenden Befunde zur Sozialpolitik in Deutschland sind vielfältig. Etliche von ihnen stützen die These von der Sozialpolitik als Stabilisierungsfaktor für Politik, Gesellschaft und Wirtschaft. Zu dieser Problemlöserfunktion gesellt sich aber eine Problemerzeugerfunktion. Diese hat mit den starken Nebenwirkungen und Folgeproblemen zu tun, die eine weit ausgebaute Sozialpolitik mit sich bringt - ebenfalls in Politik, Gesellschaft und Wirtschaft. Die Sozialpolitik ist insoweit beides: Problemlöser und Pro-

28 Statistisches Bundesamt u.a. (Hg.), Datenreport 2008, S. 294 auf der Basis der Sozialstaats-Surveys von 2005 und 2006.

29 Statistisches Bundesamt u.a. (Hg.), Datenreport 2008, S. 293.

30 Die Zitate stammen aus einem Diskussionsbeitrag von Oskar Niedermayer auf der Podiumsdiskussion vom 22.9.2009 im Rahmen des Kongresses der Deutschen Vereinigung für Politische Wissenschaft in Kiel. 
blemerzeuger, Stabilisator und Destabilisator. Aber damit nicht genug: Die vorliegenden Befunde machen zudem auf eine für die Sozialpolitiker und für die Sozialpolitik insgesamt beunruhigende Diskrepanz aufmerksam: eine größer werdende Lücke zwischen umfänglichen Sozialstaatsinvestitionen und vorzeigbaren Leistungsprofilen der Sozialpolitik bei ihren Kernaufgaben einerseits und rückläufiger Akzeptanz und abnehmender Zufriedenheit mit der Sozialpolitik andererseits.

\section{Zusammenfassung}

Wirkt die Sozialpolitik als Stabilisierungsfaktor von Politik, Gesellschaft und Wirtschaft in der Bundesrepublik Deutschland? Die vorliegenden Befunde der Forschung zeigen doppelwertige Wirkungen der Sozialpolitik an: Die Sozialpolitik hat ihre ureigenen Aufgaben - Vermeidung materieller Verelendung, Abschirmung gegen Risiken und Abbau krasser sozialer Ungleichheit - relativ erfolgreich bewältigt. Allerdings ist dafür ein hoher Preis zu entrichten, der fast ein Drittel des Bruttoinlandsproduktes ausmacht. Zudem ist die Sozialpolitik zweiwertig: sie ist Problemlöser und zugleich Problemerzeuger - in der Politik, in der Gesellschaft und in der Ökonomie. Zudem verweisen die vorliegenden Befunde auf einen abnehmenden politischen Grenznutzen der Sozialpolitik hin: auf eine größer werdende Lücke zwischen umfänglichen Sozialstaatsinvestitionen und vorzeigbaren Leistungsprofilen der Sozialpolitik bei ihren Kernaufgaben einerseits und rückläufiger Akzeptanz der Sozialpolitik sowie abnehmender Zufriedenheit mit ihr andererseits.

\section{Summary}

The literature on Germany's welfare state points to ambivalent effects of social policy. As regards its main targets, social policy has been relatively successful: it shelters the population from pauperisation, protects almost all citizens through income maintenance schemes against major social risks, and reduces massive levels of social inequality. However, the costs involved in these achievements have been considerable: Germany's social budget consumes almost one third of Gross Domestic Product. Moreover, social policy has been both a problem solver and a problem creator - in the economy, in society and in politics. Furthermore, surveys point to decreasing political returns of social policy: A gap has emerged between high investment in social policy and reasonable success in the core functions of the German welfare state on the one hand and decreasing levels of acceptance of, and growing dissatisfaction with, social policy on the other.

Manfred G. Schmidt, Has social policy stabilized Germany's political, social and economic development? 